\title{
Electrical neuroimaging during auditory motion aftereffects reveals that auditory motion processing is motion sensitive but not direction selective
}

\author{
David A. Magezi, Karin A. Buetler, Leila Chouiter, Jean-Marie Annoni, and Lucas Spierer \\ Neurology Unit, Department of Medicine, Faculty of Sciences, University of Fribourg, Fribourg, Switzerland
}

\begin{abstract}
Magezi DA, Buetler KA, Chouiter L, Annoni JM, Spierer L. Electrical neuroimaging during auditory motion aftereffects reveals that auditory motion processing is motion sensitive but not direction selective. J Neurophysiol 109: 321-331, 2013. First published October 17, 2012; doi:10.1152/jn.00625.2012.-Following prolonged exposure to adaptor sounds moving in a single direction, participants may perceive stationary-probe sounds as moving in the opposite direction [direction-selective auditory motion aftereffect (aMAE)] and be less sensitive to motion of any probe sounds that are actually moving (motion-sensitive aMAE). The neural mechanisms of aMAEs, and notably whether they are due to adaptation of direction-selective motion detectors, as found in vision, is presently unknown and would provide critical insight into auditory motion processing. We measured human behavioral responses and auditory evoked potentials to probe sounds following four types of moving-adaptor sounds: leftward and rightward unidirectional, bidirectional, and stationary. Behavioral data replicated both direction-selective and motion-sensitive aMAEs. Electrical neuroimaging analyses of auditory evoked potentials to stationary probes revealed no significant difference in either global field power (GFP) or scalp topography between leftward and rightward conditions, suggesting that aMAEs are not based on adaptation of direction-selective motion detectors. By contrast, the bidirectional and stationary conditions differed significantly in the stationary-probe GFP at $200 \mathrm{~ms}$ poststimulus onset without concomitant topographic modulation, indicative of a difference in the response strength between statistically indistinguishable intracranial generators. The magnitude of this GFP difference was positively correlated with the magnitude of the motion-sensitive aMAE, supporting the functional relevance of the neurophysiological measures. Electrical source estimations revealed that the GFP difference followed from a modulation of activity in predominantly right hemisphere frontal-temporal-parietal brain regions previously implicated in auditory motion processing. Our collective results suggest that auditory motion processing relies on motion-sensitive, but, in contrast to vision, non-directionselective mechanisms.
\end{abstract}

acoustic spatial processing; illusory motion; EEG; evoked potentials; human cerebral cortex

VISUAL MOTION AFTEREFFECTS, the illusory motion of stationary visual stimuli (probes) following exposure to a prolonged moving stimulus (adaptor), are due to adaptation of directionselective neurons within the visual motion pathway (for review, see Niedeggen and Wist 1998; Thompson and Burr 2009). By contrast, the neural basis of their auditory counterparts, the auditory motion aftereffects (aMAEs), remains unknown. Studies of aMAEs have indeed so far been limited to psychophysical investigations; they showed that prolonged exposure to adaptor sounds moving in a particular direction

Address for reprint requests and other correspondence: D. A. Magezi, Donders Institute for Brain, Cognition and Behaviour, Radboud University Nijmegen, The Netherlands (e-mail: auditory@magezi.com). induces two types of aMAEs. The "direction-selective" aMAE is the perception of a stationary probe sound moving in the opposite direction to the adaptor (Dong et al. 2000; Grantham and Wightman 1979; Grantham 1998; Neelon and Jenison 2003). The "motion-sensitive" aMAE is a loss of sensitivity to the motion, in any direction, of moving probes sounds; specifically, the minimum angle that a horizontally moving sound must traverse to be reliably perceived as moving is significantly elevated following moving adaptors (Grantham 1989, 1992). Elucidating the neural basis of aMAEs will provide important insight into the mechanisms of human auditory motion processing, which remains poorly understood. Various current models of auditory motion processing predict different patterns of brain responses to the stationary probe during the aMAEs (Fig. 1, Table 1).

A first model posits that, as in vision, auditory motion perception relies on direction-selective motion detectors (Wagner et al. 1997). According to this model, henceforth referred to as the "direction-selective model," horizontal motion perception would depend on the balance of activity between motion detectors selective for leftward or rightward motion. This is depicted schematically in Fig. $1 A$, where the relative spike rates are plotted as a function of interaural time difference (ITD) (e.g., see Malone et al. 2002) for leftward- and rightward-selective motion adaptors, shown in red and blue, respectively. The dashed red line in Fig. $1 A$ shows the increased response of the leftward motion detectors at the onset of a unidirectional leftward adaptor. During exposure to this unidirectional adaptor, selective neural adaptation will result in a reduced response for motion detectors selective for that direction (solid red line, Fig. 1A), but not for motion detectors selective for the opposite direction (solid blue line, Fig. 1A). Therefore, when the stationary probe is presented, the mismatch in response size between the leftward and rightward motion detectors (see arrows in Fig. 1A) will result in illusory rightward motion, that is, a direction-selective aMAE. Similarly, a unidirectional rightward adaptor will result in the perception of illusory leftward motion of a stationary probe due to selective adaption of the rightward motion detectors (see arrows in Fig. 1C). In this way, the brain response to a stationary probe sound would be expected to differ quantitatively between the leftward (Fig. 1A) and rightward (Fig. 1C) unidirectional adaptor conditions because of the difference in relative activity of distinct neural populations (solid red and blue lines in Fig. 1, $A$ and $C$ ).

During exposure to an alternating leftward and rightward adaptor (bidirectional condition, Fig. $1 E$ ), the responses of both leftward and rightward motion detectors will be increased at onset (dashed lines, Fig. 1E) and then reduced due to neural 


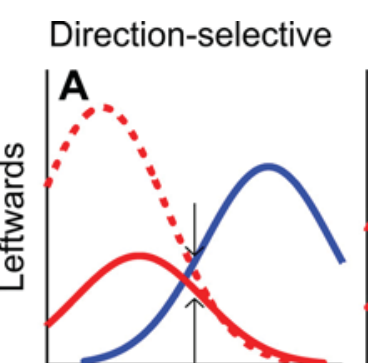

\section{Motion-sensitive}
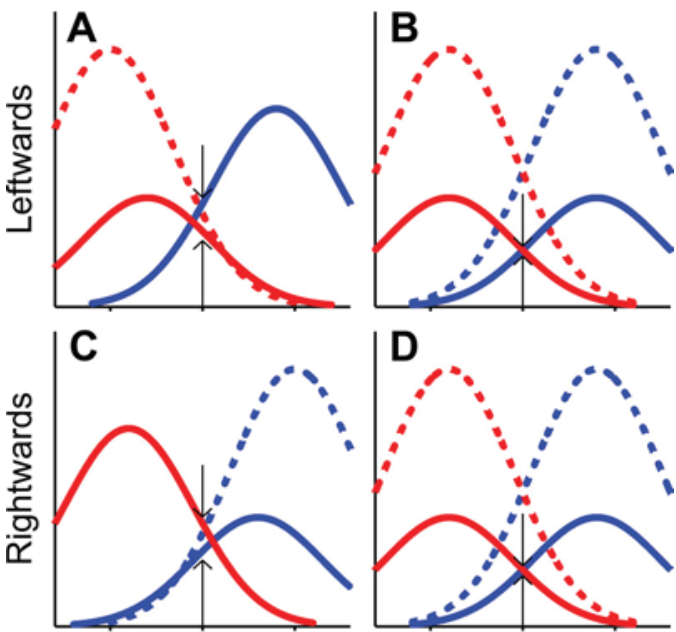

D
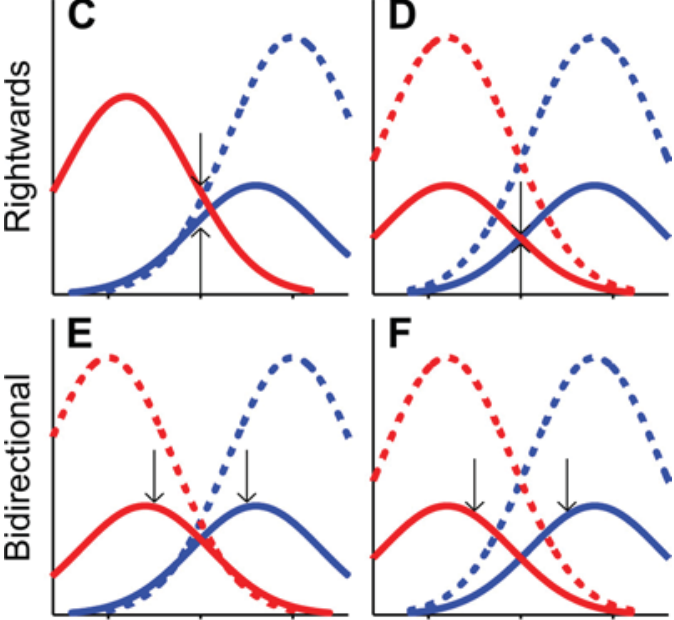

$\mathbf{F}$
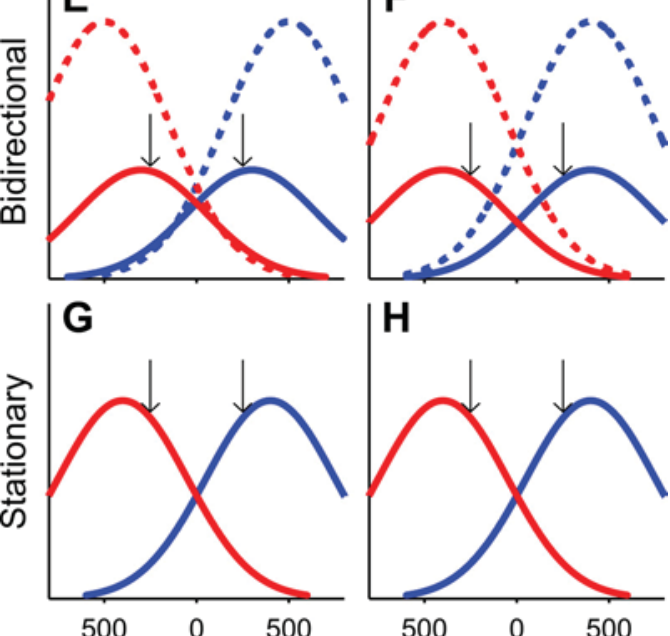

H

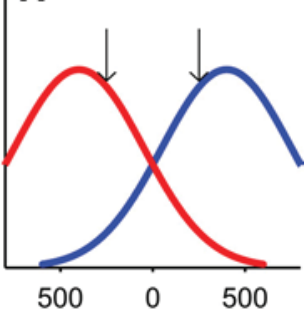

ITD in $\mu$ s

Fig. 1. Schematic representation of neural tuning curves according to directionselective (left: $A, C, E$, and $G$ ) and motion-sensitive models (right: $B, D, F$, and $H$ ). For each adaptor condition, relative spike rates are shown as a function of interaural time difference (ITD). Red and blue curves represent neural populations maximally tuned to left and right hemispace, respectively. Dashed and solid lines show activity before and after adaptation, respectively (see Introduction). Arrows indicate the maximum expected activity for each population in response to stationary $(A-D)$ or moving probes $(E-H)$.

adaptation (solid lines, Fig. 1E). This reduction in response would result in reduced sensitivity to motion in probes that are actually moving (see arrows in Fig. $1 E$ ) and would explain the behavioral motion-sensitive aMAE. In comparison, this neural adaptation, and hence reduction in perceptual sensitivity, will not occur during presentation of a stationary adaptor (stationary condition, Fig. $1 G$ ). The brain responses to any probes, including stationary, would be expected to differ quantitatively between bidirectional and stationary conditions due to a difference in the level of activity within the same neural generators (compare solid lines in Fig. 1, $E$ and $G$ ). The predictions for the direction-selective model are summarized in Table 1. 1) The brain responses to the stationary probe in the leftward ( $\mathrm{Lw}$ ) and rightward ( $\mathrm{Rw}$ ) conditions may have similar response strength (level of activity), but would be expected to differ in the configuration of neural populations ("configuration"). 2) In contrast, the brain responses to the stationary probe in the bidirectional (Bid) and stationary (Stat) conditions would be expected to differ in response strength ("strength"), but not in the configuration of neural generators.

In stark contrast to the direction-selective model, other authors have proposed that, rather than motion detectors, auditory motion perception could be based on serial "snapshots" of static sound localization (Grantham 1986; Middlebrooks and Green 1991). Because adaptation cannot occur if there are no motion detectors, this model, referred to as the "snapshot model," predicts that any behaviorally measured aMAEs would be due to other factors, such as response bias, and thus brain response to stationary probes would be the same in all four conditions: that is, no differences in response strength or configuration of neural generators (Table 1).

Finally, auditory motion perception may rely on neurons that are motion sensitive, but not direction selective (Griffiths et al. 2000; Scott et al. 2009; Spitzer and Semple 1991). According to this model, referred to as the "motion-sensitive model," during exposure to a moving adaptor, motion detectors would show the same pattern of adaptation, irrespective of whether the adaptor was unidirectional leftward (Fig. 1B), rightward (Fig. $1 D$ ), or bidirectional (Fig. $1 F$ ). On the one hand, this motion-sensitive model could not explain the direction-selective aMAE, and the brain response to stationary probes would not be expected to differ between leftward and rightward conditions (compare arrows in Fig. 1, $B$ and $D$; Table 1). On the other hand, the motion-sensitive model could explain the behavioral motion-sensitive aMAE in a similar way to the direction-selective model (compare arrows in Fig. 1, $F$ and $H$ ). Neural adaptation would result in reduced activity following the bidirectional adaptor (Fig. $1 F$ ), but not the stationary adaptor (Fig. 1H), and thus the brain responses to probes would be expected to quantitatively differ between bidirectional and stationary conditions due to a difference in the level of activity within the same neural generators (Fig. $1, F$ and $H$; Table 1).

To directly compare these models, we analyzed behavioral and electrical neuroimaging measures to probe sounds during various conditions of aMAE. Participants reported the perceived direction (left or right) and extent of motion (small or large) for probe sounds presented after four different adaptor sounds: leftward, rightward, bidirectional, and stationary. Probe sounds were either stationary or moving. We performed comparisons of auditory evoked potentials (AEPs) between the unidirectional adaptor conditions (leftward and rightward), and between bidirectional and stationary conditions, to explore the neural basis of the behavioral aMAEs. As described in METHODS, these comparisons were done for both the global field power (GFP) and global map dissimilarity (GMD), to examine differences in re-

Table 1. Qualitative predictions of three different models of auditory motion perception (see Fig. 1 and Introduction)

\begin{tabular}{llllll}
\hline \hline \multirow{2}{*}{ Model } & \multicolumn{2}{c}{ Lw vs. Rw } & & \multicolumn{2}{c}{ Bid vs. Stat } \\
\cline { 2 - 3 } \cline { 5 - 6 } & Strength & Configuration & & Strength & Configuration \\
\hline Direction-selective & Same & Different & & Different & Same \\
Snapshot & Same & Same & & Same & Same \\
Motion-sensitive & Same & Same & & Different & Same \\
\hline
\end{tabular}

Lw, leftward; Rw, rightward; Bid, bidirectional; Stat, stationary. 


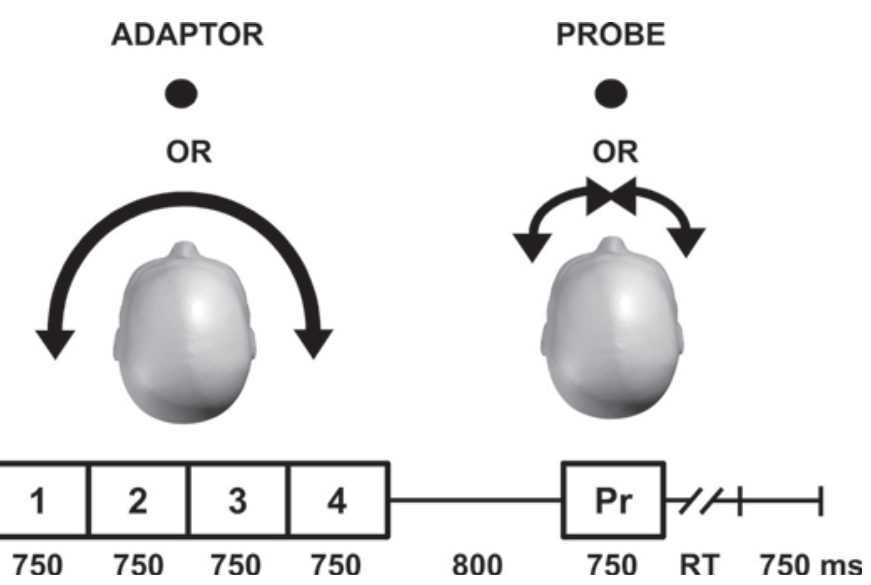

Fig. 2. Experimental paradigm. Each trial comprised a 3,000-ms adaptor followed, after $800 \mathrm{~ms}$, by a probe (Pr) (Table 3). The adaptor comprised four sections (Table 2), each of 750-ms duration. Participants had up to 2,000 ms to respond [response time (RT)], after which the next trial would commence following an intertrial interval of $750 \mathrm{~ms}$.

sponse strength (level of activity) and in the configuration of underlying neural generators, respectively, thereby allowing us to directly test the predictions of the three different models of auditory motion processing (Table 1).

\section{METHODS}

Participants. Thirteen (4 women; aged 19-38 yr, mean age $25 \pm$ $5 \mathrm{yr}$ ) took part in the study. All reported normal hearing and normal or corrected to normal vision, and none had a history of neurological or psychiatric illness. According to the Edinburgh Inventory (Oldfield 1971), 12 participants were right-handed and one was left-handed. Each participant provided written, informed consent to the procedures, which were approved by the Ethics Committee of the University of Fribourg.

Stimuli. A 3,000-ms adapting sound (referred to as the "adaptor") was followed, after a silent interstimulus interval of $800 \mathrm{~ms}$, by a 750-ms probe sound (the "probe"), as shown in Fig. 2. Both adaptor and probe were random Gaussian noise. There were four adaptor conditions with different trajectories of motion (Table 2). The auditory motion of the adaptor and probe sounds was achieved by manipulating the ongoing ITD. ITD is the most important cue for localizing, in the horizontal plane, sounds that include low frequencies (Wightman and Kistler 1992), and avoids the confounding monaural changes in level or frequency between the ears induced by other spatial cues (see DISCUSSION). In the first three conditions, the ITD of the adaptor sound was changed linearly from -500 to $500 \mu$ s, either left- (negative ITD) or right-ear leading (positive ITD), to simulate auditory motion (e.g., Ducommun et al. 2002). This motion took place over a period of $750 \mathrm{~ms}$ and was repeated four times during the 3,000-ms adaptor sound (Fig. 2). This design of adaptor ensured the presentation of

Table 2. Adaptor sounds: trajectory of motion during each section (see Fig. 2), and number of trials presented

\begin{tabular}{lccccc}
\hline \hline \multirow{2}{*}{ Adaptor } & \multicolumn{5}{c}{ Section } \\
\cline { 2 - 5 } & 1 & 2 & 3 & 4 & No. Trials \\
\hline Leftward & $\leftarrow$ & $\leftarrow$ & $\leftarrow$ & $\leftarrow$ & 240 \\
Rightward & $\rightarrow$ & $\rightarrow$ & $\rightarrow$ & $\rightarrow$ & 240 \\
Bidirectional & $\leftarrow$ & $\rightarrow$ & $\leftarrow$ & $\rightarrow$ & 120 \\
Bidirectional & $\rightarrow$ & $\leftarrow$ & $\rightarrow$ & $\leftarrow$ & 120 \\
Stationary & $\bullet$ & $\bullet$ & $\bullet$ & $\bullet$ & 240 \\
\hline
\end{tabular}

Auditory adaptors: $\leftarrow, 500$ to $-500 \mu \mathrm{s} ; \rightarrow,-500$ to $500 \mu \mathrm{s} ; \bullet, 0 \mu \mathrm{s}$. motion at an optimum velocity ( $\sim 240^{\circ} / \mathrm{s}$; Grantham and Wightman 1979; Grantham 1989) for an optimum duration (Neelon and Jenison 2004 ) to induce an aMAE, within the time constraints of an electroencephalographic (EEG) study. In the leftward and rightward conditions, the ITD during each of these periods of motion was initially leading in the right or left ear, respectively, creating the percept of motion in one direction, left- (Lw) or rightward (Rw) only, during each of the four periods. In the third condition, bidirectional, the direction of motion would alternate over the four periods: Lw-Rw$\mathrm{Lw}-\mathrm{Rw}$, or Rw-Lw-Rw-Lw. In the fourth condition, stationary, the adaptor ITD was fixed at $0 \mu$ s for all four periods, creating the perception of a stationary, midline sound.

In all four conditions, the adaptor could be followed by one of five types of probe (Table 3). For four of these probes, the ITD was changed linearly over $750 \mathrm{~ms}$. The ITD trajectories were from 0 to $-150,-150$ to 0,0 to 150 , or 150 to $0 \mu \mathrm{s}$, resulting in strong percepts of motion from midline-to-left, left-to-midline, midline-to-right, and right-to-midline, respectively. For the fifth type of probe, the ITD was held constant at $0 \mu \mathrm{s}$, and this is referred to as the "stationary probe". To ensure that all spectral components of the auditory stimuli conveyed unambiguous ITD cues (that is, component interaural phase differences were never greater than $\pi$ ), sounds were low-pass filtered at $1 \mathrm{kHz}$ using a fourth-order Butterworth filter. After filtering, all sounds were gated on and off with $5-\mathrm{ms}$ cosine ramps, which were synchronous at the two ears to avoid envelope ITD cues. Envelope ITDs are ineffective at low frequencies (Henning 1983; Henning and Ashton 1981) and may differ from ongoing ITDs in terms of the neural encoding mechanisms (Dreyer and Delgutte 2006). Within the adaptor sounds, gating was applied to each of the four periods, to avoid the percept of motion in the reverse direction at the transition between periods.

Matlab (Mathworks, Natick, MA) was used to create auditory stimuli, which were presented via headphones (Sennheiser HD428, Old Lyme, CT). Stimulus delivery and response collection were carried out with E-Prime 2.0 (Psychology Software Tools, Sharpsburg, PA) and a 22-inch LED monitor (Samsung BX2250; 1,920 $\times$ 1,080 pixel resolution; $60-\mathrm{Hz}$ frame rate) for the fixation-cross (see below).

Procedure. Each trial comprised an adaptor followed by a probe sound (Fig. 2). Participants were asked to judge the direction (leftward or rightward) and the extent (small or large) of motion of the probe sounds by pressing one of four response buttons arranged in the following order (from left to right): leftward-large extent, leftward-small extent, rightward-small extent, and rightward-large extent. Responses were made with the same hand throughout the experiment. Participants had up to $2,000 \mathrm{~ms}$ to respond, and no feedback was given. After each response, the next trial started after an intertrial interval of $750 \mathrm{~ms}$. To reduce the influence of eye movements, participants were asked to fixate a cross presented in the center of the screen from the beginning of the adaptor until the end of the probe sound.

To reduce fatigue, the experiment was divided into blocks. Each block comprised 60 trials and lasted $\sim 7$ min. There were 16 blocks ( 4 blocks per adaptor condition). Participants had a break of at least 1 min between each block. The adaptor condition was kept the same for four consecutive blocks $(4$ blocks per condition $\times 60$ trials per block $=240$ trials per

Table 3. Probe sounds: motion trajectory and probability of presentation

\begin{tabular}{lrcc}
\hline \hline Probe Direction & Start ITD, $\mu \mathrm{s}$ & End ITD, $\mu \mathrm{s}$ & Probability, $\%$ \\
\hline Midline-to-left & 0 & -150 & 5 \\
Left-to-midline & -150 & 0 & 5 \\
Midline-to-right & 0 & 150 & 5 \\
Right-to-midline & 150 & 0 & 5 \\
Stationary* & 0 & 0 & 80 \\
\hline
\end{tabular}

*Auditory evoked potentials were only analyzed for the stationary probe. 
condition; Table 2). After these four consecutive blocks, there was a break of at least $5 \mathrm{~min}$ before a new adaptor condition was presented to prevent "carry on" of adaptation effect. The probability of a stationary probe was $80 \%$ (Table 3). In this way, for each adaptor condition, there were on average $192(=80 \% \times 240$ trials per condition $)$ stationary-probe trials, which were the focus of the EEG analysis.

Analysis of behavioral data. For each condition, the direction of the aMAE was measured using a direction-selectivity index calculated by subtracting the number of "leftward" from the number of "rightward" responses, divided by the total number of responses. Values of -1 or 1 indicate that all probes were perceived as moving leftward or rightward, respectively.

For each condition, the amplitude of the aMAE was measured using a motion-sensitivity index calculated by subtracting the number of responses for "small" from the number of responses for "large" extents of motion, divided by the total number of responses. Values of -1 or 1 indicate that all probes were perceived as having a small or large extent of motion, respectively.

The direction-selective aMAE would manifest as a difference in direction-selectivity index between leftward and rightward adaptor conditions, while a motion-sensitive aMAE would manifest as a difference in motion-sensitivity index between bidirectional and stationary conditions.

Paired $t$-tests were conducted to analyze the difference in directionselectivity or motion-sensitivity index across conditions. It is important to note that all probes were included in the behavioral analysis, although only the stationary probes were included in the EEG analysis (Table 3).

EEG data acquisition and preprocessing. Continuous EEG was acquired at $1,024 \mathrm{~Hz}$ through a 128-channel Biosemi ActiveTwo system. Preprocessing was performed using FieldTrip (Oostenveld et al. 2011), which runs under Matlab. The data were 1) resampled at $512 \mathrm{~Hz}$ to reduce computation time; 2) band-pass filtered from 0.1 to $40 \mathrm{~Hz}$ by applying a second-order zero-phase Butterworth filter in both forward and reverse time order, and notch filtered at $47-53 \mathrm{~Hz}$; and 3 ) divided into stimulus-locked epochs covering the period -150 to $600 \mathrm{~ms}$ relative to probe sound onset (385 time frames per epoch). Only epochs for the stationary-probe sounds were included in the EEG analysis (Table 3). The EEG epochs were averaged, for each participant, for the leftward, rightward, bidirectional, and stationary adaptor conditions. In addition to a $\pm 100-\mu \mathrm{V}$ artifact rejection criterion, EEG epochs containing eye blinks or other noise transients were removed after visual inspection. Data were baseline corrected using the mean over the whole epoch. The average number $( \pm \mathrm{SE})$ of accepted epochs was $165 \pm 4,161 \pm 6,164 \pm 7$, and $165 \pm 4$ for the leftward, rightward, bidirectional, and stationary conditions, respectively. Paired comparisons showed that there were no significant differences in the number of accepted epochs between leftward and rightward $[t(12)=0.90, P=0.384]$ or between bidirectional and stationary conditions $[t(12)=-0.18, P=0.854]$, ensuring that any difference in the contrasted AEPs did not follow from differences in the signal-to-noise ratio between conditions.

Artifact electrodes from each participant were then interpolated (Perrin et al. 1987) using EEGLab (Delorme and Makeig 2004), which runs under Matlab. The mean percentage $( \pm \mathrm{SE})$ of interpolated electrodes was $2.9 \pm 1.3,2.7 \pm 0.9,2.7 \pm 1.2$, and $3.0 \pm 1.2 \%$ for the leftward, rightward, bidirectional, and stationary conditions, respectively.

EOG data acquisition and analysis. To assess for the possible influence of eye position, electrooculography (EOG) was acquired from four participants using three additional electrodes, which were placed above the nasion and below the outer canthi of the eyes (e.g., see Schlögl et al. 2007). EOG data were 1) acquired at $1,024 \mathrm{~Hz}$ simultaneously with the EEG data via the Biosemi ActiveTwo system; 2) band-pass filtered from 0.1 to $100 \mathrm{~Hz}$ by applying a second-order zero-phase Butterworth filter in both forward and reverse time order, and notch filtered at $47-53 \mathrm{~Hz}$; and 3) divided into stimulus-locked epochs covering the period -150 to $600 \mathrm{~ms}$ relative to probe sound onset (385 time frames per epoch). Epochs for the stationary probe sound were averaged for each adaptor condition and submitted to a repeated-measures ANOVA with within-participant factors of EOG electrode and adaptor condition. There was no main effect of condition $[F(3,9)=1.42, P=0.301]$, and no interaction $[F(6,18)=1.29$, $P=0.309]$, which indicates that mean eye position did not significantly differ between the conditions.

Global electrical field analyses. To investigate the neural correlates of the direction-selective aMAE, AEPs to stationary probes were compared between the leftward and rightward adaptor conditions. To investigate the neural correlates of the motion-sensitive aMAE, stationary-probe AEPs were compared between bidirectional and stationary adaptor conditions. These comparisons were based on referenceindependent measures of the strength and of the topography of the electrical field of scalp potentials. These analyses of event-related potentials have been detailed elsewhere and provide several benefits over canonical AEP waveform analyses (Murray et al. 2008; Tzovara et al. 2012). In particular, they circumvent the potential biases induced by a priori selection of restricted electrodes or time periods of interest, and they enable data-driven, statistically based neurophysiological interpretations of AEP modulations.

Modulations in the strength of the electric field at the scalp were assessed using the GFP (Lehmann and Skrandies 1980), which is calculated as the spatial standard deviation of the potentials at all electrodes at each time frame. Modulations in the topography of scalp potentials were assessed using the GMD, which is equivalent to the spatial Pearson's product-moment correlation coefficient between the potentials of the two maps being compared (e.g., Murray et al. 2008).

GFP difference and GMD were analyzed at each time frame using randomization statistics (Koenig et al. 2011), where values at each time frame were compared with an empirical distribution derived from bootstrapping (5,000 permutations). GFP difference and GMD were calculated between the leftward and rightward conditions, and between the bidirectional and stationary conditions.

GFP and GMD are orthogonal measures, and the combinations of modulations at the level of one or both of these measures provide a direct neurophysiological interpretability: a topographic modulation with or without concomitant GFP modulation necessarily follows from a change in the configuration of the underlying intracranial generator (Lehmann and Skrandies 1980). Therefore, a modulation in response strength (as indexed by the GFP) without concomitant topographic modulation can be interpreted as a change in the strength of the response of statistically indistinguishable generators (Murray et al. 2008).

Effects were considered reliable if they met or exceeded the $P<0.05$ criterion. Multiple comparisons and temporal auto-correlation were corrected by application of a >11-contiguous data point temporal criterion (Guthrie and Buchwald 1991). Guthrie and Buchwald (1991) generated pseudorandom temporally autocorrelated EEG data sets to show that, when comparing across conditions, the number of continuous significant data points that would be expected to arise by chance (based on 1,000 permutations) varies as a function of the number of data points in the epoch, and to a much lesser extent the number of participants and the temporal criterion level chosen $(P<0.05$ or $P<0.1$ in their simulations). This approach for dealing with the temporal auto-correlation found in EEG data is one of the recommended approaches in authoritative guidelines on EEG analysis (e.g., Picton et al. 2000) and has been incorporated into a number of other analysis methods (e.g., Blair and Karniski 1993, Maris and Oostenveld 2007). We applied the technique to our data set (13 participants, 385 time points) and showed that number of significant contiguous data points was $>11$ and $>15$ for the temporalcriterion levels of $P>0.05$ and $P>0.1$, respectively. Both of these criterion levels were examined to avoid the cliff effect of 0.05 (Rosenthal and Gaito 1963; Rosnow and Rosenthal 1989), but as there was no difference in the pattern of results, only the results for $P>0.05$ are reported. 
Electrical source estimation. Electrical sources underlying the scalp-recorded data were estimated for the period of significant difference found in the global electrical field analyses. This was done using a distributed linear inverse solution based on a local autoregressive average (LAURA) regularization approach (Grave de Peralta et al. 2001; Grave de Peralta et al. 2004; see also Michel et al. 2004 for a comparison of inverse solution methods), which was run in Cartool software (Brunet et al. 2011). LAURA selects the source configuration that mimics the biophysical behavior of electric fields (i.e., activity at one point depends on the activity at neighboring points). The solution space is based on a realistic head model and included 3,005 solution points homogeneously distributed within the gray matter of the average brain of the Montreal Neurological Institute (courtesy of R. Grave de Peralta Menendez and S. Gonzalez Andino, University Hospital of Geneva, Geneva, Switzerland). Intracranial sources were statistically compared at each node level between conditions using the same within-subject designs as in the global electrical field analyses.

Electrode-level analyses. Global field analyses described above are based on the activity of all scalp electrodes. Due to the absence of significant GMD (see RESULTS), we also performed more spatially sensitive analyses at the electrode level. To correct for multiple comparisons, we used a nonparametric randomization method (Maris and Oostenveld 2007) to identify clusters of electrodes which differed significantly between the conditions being compared. Clusters were identified as spatially contiguous electrodes where the $t$ statistics exceeded a threshold $(P<0.05)$, and the cluster-level statistic was defined as the sum of the $t$ statistics of the electrodes in that cluster. To control for the type I error rate for the complete set of 128 electrodes, the data were randomly permuted between the two adaptor conditions within every participant, and a reference distribution of the cluster-level $t$ statistic was created from 1,000 random sets of permutations. The nonparametric $P$ value was estimated as the proportion of permutations in this distribution, which exceeded the observed maximum cluster-level statistic.

\section{RESULTS}

Behavioral results: direction-selective aMAE. Participants showed a direction-selective aMAE. There was a significant difference in direction-selectivity indexes for stationary probes between the leftward and rightward adaptor conditions $[t(12)=4.15, P=0.001]$, indicating that, following an adaptor moving in a single direction (leftward and rightward conditions), stationary probes were perceived as moving in the opposite direction (Fig. 3A). On average, the direction of moving probes was accurately perceived in all conditions: direction-selectivity indexes $( \pm \mathrm{SE})$ were $-0.7 \pm 0.2$ and $0.6 \pm 0.2$ for leftward and rightward moving probes, respectively (see vertical dotted lines in Fig. $3 A$ ).

Behavioral results: motion-sensitive aMAE. Participants also demonstrated a motion-sensitive aMAE. Motion-sensitivity indexes to moving probes were smaller in the bidirectional than the stationary adaptor conditions $[t(12)=-5.10, P<$ $0.001]$, indicating that moving probes were perceived as having a larger extent of motion when presented after a stationary adaptor than after a moving adaptor (Fig. 3B). Stationary probes were, on average, perceived as having a small extent of motion in all conditions: motion-sensitivity indexes of $-0.7 \pm$ 0.1 (vertical dotted line in Fig. $3 B$ ).

AEPs: direction selectivity. Stationary probe sounds elicited robust auditory evoked responses (AEPs), which had a typical AEP waveform morphology (Fig. 4A). Directional selectivity in the electrical brain response to stationary probe sounds was

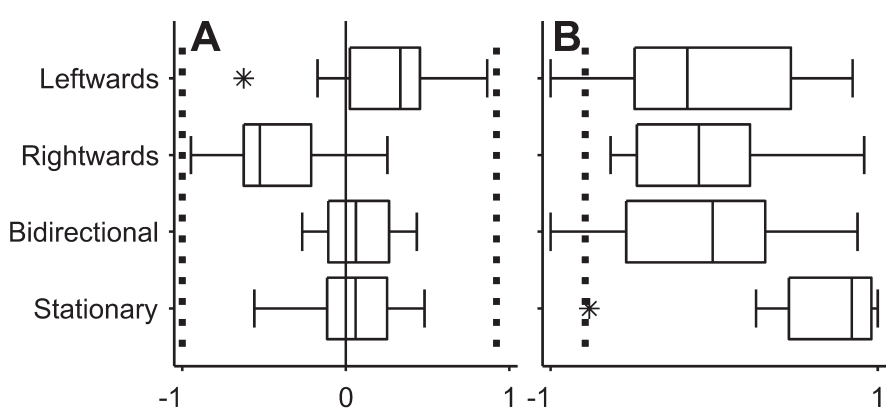

Direction-selectivity ind. Motion-sensitivity ind.

Fig. 3. Behavioral responses in the four adaptor conditions. A: directionselectivity indexes. Data for stationary probes are shown as box-and-whisker plots, while vertical dotted lines near -1 and +1 show the median (across conditions) for the leftward and rightward moving probes, respectively. A direction-selective auditory motion aftereffect (aMAE) was present (see RESULTS). $B$ : motion-sensitivity indexes. Data for moving probes are shown as box-and-whisker plots, while the vertical dotted line shows the median for the stationary probes. There was a motion-sensitive aMAE. The boxes are composed of lines at the lower quartile, median, and upper quartiles values; whiskers represent 1.5 times the h-spread (range of scores between the hinges), and outliers are shown as asterisks.

analyzed by contrasting probe AEPs in the leftward vs. rightward adaptor conditions. This analysis showed that there was no statistically significant difference $(P<0.05,>11$ data points) in scalp topography (GMD, Fig. $4 C$ ). Importantly, this was still the case when the analysis was restricted to only include AEPs of trials with a behavioral direction-selective aMAE; that is, where the stationary probes were perceived as moving in the direction opposite to that of the adaptor (thin blue lines in Fig. $4 C$ ). To increase spatial sensitivity, a comparison between leftward and rightward conditions was also performed at the electrode level, using cluster-based permutation technique to correct for multiple comparisons (see METHODS). No significant clusters were found. Similarly, there was no significant difference in the GFP between leftward and rightward conditions (GFP, Fig. 4B).

AEPs: motion sensitivity. Motion sensitivity in the electrical brain response to stationary probes was analyzed by contrasting probe AEPs in the bidirectional vs. stationary adaptor conditions. There was a significant difference $(P<0.05,>11$ data points) in GFP between the two conditions from 188 to $211 \mathrm{~ms}$ poststimulus onset (Fig. 4D). This was due to a significantly higher mean GFP in the bidirectional than the stationary condition over this period [Fig. $4 F ; t(12)=2.34$, $P<0.05]$. This difference in GFP was positively correlated with the difference in the corresponding motion-sensitivity indexes (Fig. 3B) for the two conditions [Pearson $r(11)=0.56$; $P<0.05$; Fig. 4D]. Source estimations revealed that the GFP difference followed from a modulation of activity $(P<0.05)$ in predominantly right-hemisphere, bilateral temporo-frontoparietal cortexes and insula (Fig. $4 H$ ). The most significant modulation was in the right inferior frontal gyrus [Brodmann Area 9, Talairach coordinates (51, 20, 24); Talairach and Tournoux, 1988]. Other regions of significant modulation included right middle frontal gyrus, bilateral insula, precentral gyrus and inferior parietal lobule, and a small part of the left superior temporal gyrus. There was no significant difference in scalp topography (GMD) between bidirectional and stationary conditions (Fig. 4E). 

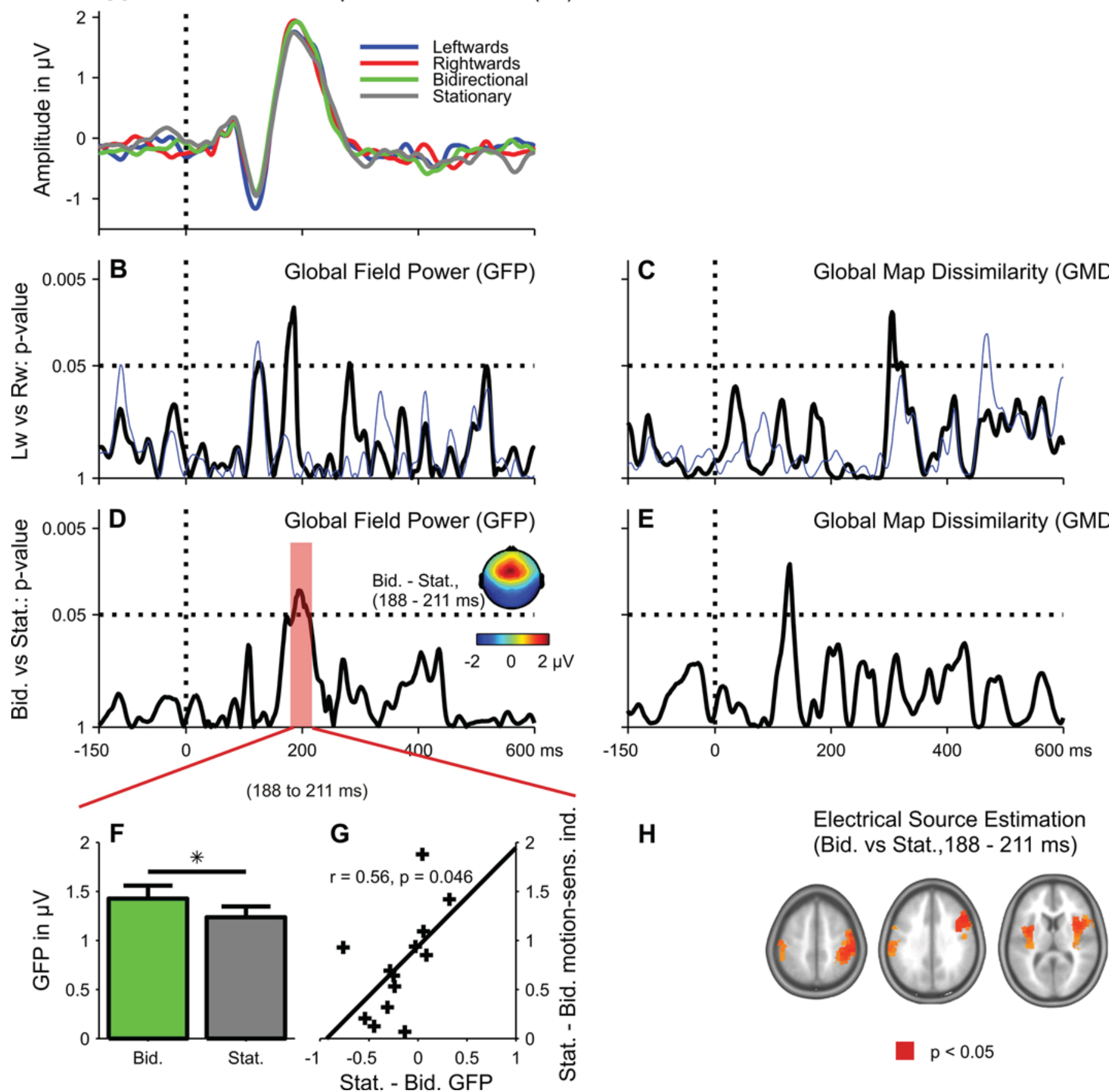

$\mathbf{H}$

Electrical Source Estimation

(Bid. vs Stat.,188 - $211 \mathrm{~ms}$ )

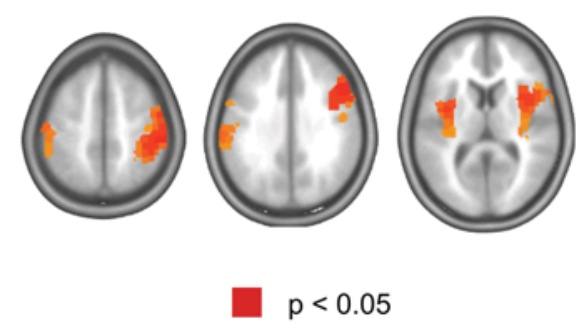

Fig. 4. Electrical brain responses. A: grand-average auditory evoked potentials (AEPs) to stationary probes at the vertex (Cz) shown for the four adaptor conditions. $B-C$ : global field analyses for leftward ( $\mathrm{Lw})$ and rightward (Rw) conditions. AEPs were compared with test for modulation in global field power $(B)$ or scalp topography [global map dissimilarity (GMD); $C$ ]. The thin blue lines show the results when only trials with a direction-selective aMAE were included. $D-E$ : global field analyses for the comparison of bidirectional (Bid) and stationary (Stat) conditions. $D$ : there was a significant GFP difference from 188 to 211 $\mathrm{ms}$. $D$, inset: the scalp distribution of difference between bidirectional and stationary conditions during this time period (188 and $211 \mathrm{~ms}) . F$ : the mean activity over the period of significant GFP difference in $D$ shows larger GFP in the bidirectional than the stationary condition. $G$ : within-participant difference in GFP (stationary-bidirectional) was correlated with the magnitude of the motion-sensitive aMAE (stationary-bidirectional motion sensitivity index). Motion-sens ind, motion-sensitivity index. $H$ : source estimates (see METHODS) for the GFP difference shown in neurological convention (right cerebral hemisphere is displayed on the right). Stimulus onset is represented by vertical dotted line at $0 \mathrm{~ms}$ in $A-E$.

\section{DISCUSSION}

We identified, for the first time, the neural correlates of the aMAEs. Behavioral results replicated previous psychophysical literature by showing a direction-selective aMAE (i.e., the perception of illusory motion of a stationary probe sound in the opposite direction to the adaptor sound), as well as a motionsensitive aMAE (a loss of sensitivity to the auditory motion of actually moving probe sounds following exposure to a moving adaptor). Comparisons between electrical neuroimaging analyses of the AEPs to the stationary probe revealed no significant 
difference in the GFP or scalp topography between the leftward and rightward adaptor conditions, even with more spatially sensitive electrode-level analyses. This pattern of results indicates that aMAEs are not due to adaptation of directionselective motion detectors, because a differential adaptation of direction-selective motion detectors (Fig. 1, $A$ and $C$ ) would have resulted in the engagement of distinct configurations of intracranial generators (direction-selective model in Table 1) and consequently in a topographic modulation (e.g., Vaughan 1982; for review, see Murray et al. 2008).

In contrast, electrophysiological responses to stationary probes in the bidirectional and stationary conditions differed significantly in GFP ( 200-ms poststimulus onset), but not in topography. This pattern of results is consistent with the motion-sensitive model (Fig. 1, $B, D, F$, and $H$ ), holding that the motion-sensitive aMAE is due to adaptation of non-direction-selective motion detectors. GFP modulation without concomitant topographic modulation indeed indicates a difference in response strength between statistically indistinguishable neural generators (e.g., Koenig and Melie-García 2010; Skrandies 2007), as predicted for the motion-sensitive model in Table 1. Further supporting this hypothesis, the GFP difference was positively correlated with the magnitude of the perceived motion-sensitive aMAE, and source estimations localized the GFP modulation within a predominantly right-lateralized, fronto-temporal-parietal network, which has previously been shown to be sensitive to sound motion (Baumgart et al., 1999; Bremmer et al. 2001; Ducommun et al. 2002; Getzmann 2011; Griffiths et al. 1994; 1998, 2000; Krumbholz et al. 2005; Lewis et al. 2000; Pavani et al. 2002; Warren et al. 2002; Xiang et al. 2002; Zimmer and Macaluso 2009).

Caution must be taken in making conclusions about the presence of a statistically significant effect for one comparison (bidirectional vs. stationary) but not for another (leftward vs. rightward). The former result can be considered a significant main effect of the factor "motion" (present or absent), whereas the latter result could be described as the absence of a main effect of the factor "direction" (leftward or rightward). A significant interaction term is necessary to conclude an interaction between factors, that is, on the difference between differences, in two factorial designs (Niewenhuis et al. 2011). However, in the present experiment, we were not able to use such an ideal $2 \times 2$ factorial design due to the impossibility of creating an auditory stimulus which has direction but lacks motion.

The magnitude and interparticipant variability of the direction-selective aMAE were comparable to previous studies (Grantham and Wightman 1979, Grantham 1989, 1998). In the seminal study by Grantham and Wightman (1979), the direction-selectivity indexes of the three participants varied between 0.65 and 1.00 , and between -0.17 and -1.00 for leftward and rightward adaptor conditions, respectively (Fig. 4 of Grantham and Wightman, 1979; compare with interquartile ranges in Fig. $3 A$ of present study). In comparison, direction-selective visual motion aftereffects often show a direction-selectivity index of magnitude 1 for all participants (for review, see Anstis et al. 1998; Mather et al. 1998, 2008). In contrast to vision, the direction-selective aMAE may not reflect genuine perception of countermotion (Ehrenstein 1994), and thus may not reflect adaptation of direction-selective motion detectors. An explanation for the perception of auditory-motion direction and the direction-selective aMAE, without direction-selective motion detectors, was proposed by Boucher et al. (2004), who advanced that auditory motion perception relies on a high-level motion system equivalent to the "third-order" motion system in vision, which is based on attentional tracking of features within a salience map (Lu and Sperling 1995, 2001). Such a system is heavily influenced by attention, and direction-selective aMAE could arise because previous motion or perceived static location (Ehrenstein 1994) acts as an invalid cue (Stanley and Mathews 2003), leading to a response bias (Grantham 1989; Neelon and Jenison 2004). The contrast between electrical neuroimaging responses to the stationary probe between the leftward and rightward conditions revealed no significant GFP or topographic modulations, even when only the AEPs associated with a direction-selective aMAE were included. Thus, although negative result should be interpreted with caution (see below), if the direction-selective aMAE was due to adaptation of direction-selective motion detectors, it would have been reflected in a difference in scalp topography between the leftward and rightward conditions during our time period of interest. Higher-order processes, such as those supporting a putative third-order motion process, are likely to have manifested at latency later than $600 \mathrm{~ms}$, because they result in motion that is similar to imagined visual motion (Boucher et al. 2004), which does not necessarily begin at stimulus onset.

There are possible alternative explanations for the negative AEP result; for example, the EEG method may not be sufficiently sensitive. To asses this, the current EEG findings should be compared with other methods that may be more sensitive, such as single-unit recordings, although the authors are not aware of single-unit studies of the aMAE. It is possible that there was a difference in the stationary-probe brain response between leftward and rightward conditions (that is, a "direction-selective" effect), which was not revealed in this study due to lack of sensitivity. It should be noted, however, that the fact we found a significant modulation of GFP in the bidirectional vs. stationary comparison suggests that the present study was sufficiently powered, and that any missed effect would likely be of very small magnitude. In agreement with this, single-unit recordings have shown that mammalian neurons which respond selectively to the direction of ITD change are very rare or absent (Altman et al. 1968, 1970; Kuwada et al. 1979; Scott et al. 2009; Spitzer and Semple 1993; Yin and Kuwada 1983). This is in stark contrast to the visual system where the majority of neurons responding to visual motion have been shown to be highly direction-selective (Albright 1984; Maunsell and van Essen 1983; Weliky et al. 1996). Although caution must be taken in inferring the functional significance of the small/ absent direction-selective neurophysiological effect in the auditory system, the present data appear to be consistent with the hypothesis that motion processing is fundamentally different between visual and auditory modalities.

Another alternative explanation for the negative AEP result in the leftward vs. rightward comparison is the exclusive use of interaural temporal delays (ITDs; see METHODS). In natural environments ITDs, interaural level differences (ILDs), as well as spectral cues (head-related transfer functions, Doppler effect) are available for auditory motion processing (e.g., Neelon and Jenison 2003; for review, see Blauert 1997), and realistic acoustic motion cues have been shown to enhance both behavioral and electrical brain responses (Getzmann and Lewald 
2010). Sound motion in natural environments would be expected to activate not only "specialized" direction-selective motion detectors, but also "non-motion-specialized" neurons, which respond to changes in sound level or frequency independent of motion. For example, due to asymmetries in the ascending auditory pathway (contralateral vs. ipsilateral projections), ILDs would be expected to result in "directionselective" responses in cortical neurons, which are sensitive to sound level. To avoid such confounds introduced by level and frequency changes, only ITD was used in the present study. Studies that found direction-selective responses with free-field stimuli (Ahissar et al. 1992; Sovijärvi and Hyvärinen 1974), head-related transfer functions (Alink et al. 2012), or ILD cues (Mäkäla and McEvoy 1996; Stumpf et al. 1992; Toronchuk et al. 1992) may not have been able to distinguish motionspecialized from nonspecialized activity. Comparison with previous studies suggests that it is unlikely that the present negative results are simply due to the use of ITD-only motion cues. First, ITD is the most important cue for horizontal localization of sounds which contain energy below $\sim 1.5 \mathrm{kHz}$; (see METHODS; Wightman and Kistler 1992), and the behavioral data replicated the findings found with free-field stimuli (e.g., Grantham and Wightman 1979). Second, the direction-selective motion detectors which underlie the visual motion aftereffect show the same direction-selectivity for physically different visual motion cues (for review, see e.g., Mather et al. 1998). If similar direction-selective motion detectors were present in the auditory system, they would be expected to respond to perceptually salient motion induced by ITD cues. Third, lesion studies with free-field stimuli have failed to find evidence for specialized direction-selective auditory motion detectors in mammalian cortex (Schreiner et al. 2012).

In addition to a behavioral direction-sensitive aMAE, we found a motion-sensitive aMAE: moving probes were perceived as having a larger range of motion in the stationary than the bidirectional condition. The aMAEs are unlikely to be due to variations in eye position, which has been shown to influence auditory spatial processing (Lewald and Ehrenstein 1996), because there was no significant difference in mean eye position between the adaptor conditions (see METHODS).

The AEPs to the stationary probe in the bidirectional and stationary adaptor conditions differed in GFP, but not topography, indicating that the two conditions differed in the level of activity within statistically indistinguishable configurations of neural generators (Koenig and Melie-García 2010; Skrandies 2007). In interpreting this result, it is important to consider not only the direction of adaptor sound motion, but also the spatial locations traversed by the adaptor and probe. First, the ITD at the end of the bidirectional adaptor (either -500 or $+500 \mu \mathrm{s}$ ) was different from that at the end of the stationary adaptor $(0$ $\mu \mathrm{s})$ by $500 \mu \mathrm{s}$. However, it seems unlikely that this contributed to the measured difference in GFP, since the corresponding final ITD between the leftward $(-500 \mu \mathrm{s})$ and rightward $(+500 \mu \mathrm{s})$ was even larger $(1,000 \mu \mathrm{s})$. Second, the overlap in static spatial location between the stationary adaptor and the stationary probe could be interpreted as a limitation of the present study, because adaptation of spatial-selective brain activity could explain why the GFP was lower following the stationary than the bidirectional adaptor, without needing to refer to an adaptation of motion-sensitive neural populations. Future studies could explore the effect of reducing this limita- tion by using a more spatially "diffuse" stationary probe (with lower interaural correlation) or stationary adaptor with nonfixed location (Getzmann and Lewald 2011), although these solutions may introduce other confounds related to the cortical processing of "diffuse" sounds (e.g., Chait et al. 2005). Importantly, even if some of the GFP difference is due to spatial selectivity (to static location), that does not rule out the presence of motion sensitivity because the presence of spatially selective responses is typical for auditory motion-sensitive neurons in the inferior colliculus (McAlpine et al. 2000; Spitzer and Semple 1993) and auditory cortex (Malone et al. 2002; Scott et al. 2009; see also Getzmann and Lewald 2011). Moreover, the magnitude of the GFP difference was correlated with the size of the motionsensitive aMAE, further supporting that the GFP modulation is a neural correlate of the motion-sensitive aMAE.

The latency of the GFP difference found in the present study (200-ms poststimulus onset) corroborates the model of Ducommun et al. (2002) on processing of actual auditory motion. They compared AEPs in lateralization (static location) and motion discrimination tasks. Importantly, they found that the topography did not differ in the first $250 \mathrm{~ms}$ and suggested that "early auditory motion processing is distinguished by amplitude modulations of the AEP." The GFP-difference latency found in the present study overlaps with processing phases sensitive to the onset of motion in an otherwise continuous sound (motion-onset response). The magnitude of the motion-onset response around $200 \mathrm{~ms}$ postmotion onset has been shown to be related to the velocity of motion (Getzmann 2009), the history of previous motion (Grzeschik et al. 2010), whether motion is toward or away from the midline (Getzmann 2011; see also Magezi and Krumbholz 2010), and attentional state (Kreitewolf et al. 2011). Although it appears that there was a significant difference in topography at $125 \mathrm{~ms}$ (GMD, Fig. 4E), which preceded the difference in GFP, the GMD result was not of significant duration (see METHODS) and thus cannot be interpreted as a genuine effect.

Source estimations revealed that the difference in GFP was due to a modulation of the activity in predominantly rightlateralized fronto-temporal-parietal cortexes and insula, which have previously been shown to be sensitive to auditory motion in human neuroimaging (Baumgart et al. 1999; Bremmer et al. 2001; Ducommun et al. 2002; Getzmann 2011; Griffiths et al. 1994, 1998, 2000; Krumbholz et al. 2005; Lewis et al. 2000; Pavani et al. 2002; Poirier et al. 2005; Warren et al. 2002; Xiang et al. 2002; Zimmer and Macaluso 2009) and lesion studies (Ducommun et al, 2004; Griffiths et al. 1996, 1997; Lewald et al, 2009). One limitation of these previous studies, however, is the use of actually moving auditory stimuli, which would necessarily activate neural populations sensitive to different (static) spatial locations (Smith et al. 2004, 2007). The present study circumvents this caveat by contrasting the AEPs to identical stationary probes and thus provides strong evidence for a neural correlate of auditory motion perception, in the absence of changing spatial location or any other confound induced by the contrast between physically differing stimuli. The source estimates in the present study suggest that predominantly frontal and parietal areas are involved in this auditory motion perception. However, in contrast to many of the previous studies, which compared brain responses to actually moving with static auditory stimuli (e.g., Krumbholz et al. 2005; Warren et al. 2002), there was very little significant modulation of activity within the posterior superior temporal 
gyrus (pSTG) or planum temporale (PT). Although caution must be taken in comparing across studies that differed in stimuli, tasks, and physiological measures, the present result would suggest that activity in the pSTG/PT is most strongly related to the physical characteristics of the stimulus, whereas the frontal and parietal activity is more strongly related to the perception of auditory motion. In support of this, in many of the studies that showed inferior frontal activation in response to auditory motion, participants performed a task that required attending to the spatial attributes of the sounds (Ducommun et al. 2002; Poirier et al. 2005; Warren et al. 2002; see, however, Getzmann 2011), while in many of the studies where no inferior frontal activation was reported, participants did not perform an auditory task (Bremmer et al. 2001; Griffiths et al. 1994, 1998; Krumholz et al. 2005; Pavani et al. 2002; see, however, Zimmer and Macaluso 2009). These observations would be consistent with the proposal that auditory spatial processing in the primate cortex takes place along a dorsal "where" stream, which involves projections from the pSTG/PT to the parietal cortex, and from parietal cortex to frontal areas (Rauschecker and Tian 2000; for review, see Rauscheker and Romanski 2011).

Understanding the neural basis of the aMAEs has critical implications for our understanding of auditory motion-processing in humans. The present study reveals that auditory motion perception is based neither on a direction-selective motion detector mechanism, nor on a purely serial "snap-shot" analysis of static location (Grantham 1997). Instead, auditory motion perception relies on motion-sensitive neural populations, which, in sharp contrast to vision, are not direction selective.

\section{ACKNOWLEDGMENTS}

We would like to thank Michaël Mouthon for technical assistance with EEG recordings and David Souto for assistance with stimulus generation. Cartool software (http://brainmapping.unige.ch/Cartool.htm) has been programmed by Denis Brunet, from the Functional Brain Mapping Laboratory, Geneva, Switzerland, and is supported by the Electroencephalography Brain Mapping Core of the Center for Biomedical Imaging (http://www.cibm.ch) of Geneva and Lausanne.

Present address of D. A. Magezi: Donders Institute for Brain, Cognition and Behaviour, Radboud University Nijmegen, The Netherlands.

\section{GRANTS}

This work was supported by a Swiss National Science Foundation grant to JMA (no. 32-138497).

\section{DISCLOSURES}

No conflicts of interest, financial or otherwise, are declared by the author(s).

\section{AUTHOR CONTRIBUTIONS}

Author contributions: D.A.M. and L.S. conception and design of research; D.A.M., K.A.B., and L.C. performed experiments; D.A.M. and L.S. analyzed data; D.A.M. and L.S. interpreted results of experiments; D.A.M. and L.S. prepared figures; D.A.M. drafted manuscript; D.A.M. and L.S. edited and revised manuscript; D.A.M., J.-M.A., and L.S. approved final version of manuscript.

\section{REFERENCES}

Ahissar M, Ahissar E, Bergman H, Vaadia E. Encoding of sound-source location and movement: activity of single neurons and interactions between adjacent neurons in the monkey auditory cortex. J Neurophysiol 67: 203215, 1992.

Albright TD. Direction and orientation selectivity of neurons in visual area MT of the macaque. J Neurophysiol 52: 1106-1130, 1984.
Alink A, Euler F, Kriegeskorte N, Singer W, Kohler A. Auditory motion direction encoding in auditory cortex and high-level visual cortex. Hum Brain Mapp 33: 969-978, 2012.

Altman JA. Are there neurons detecting direction of sound source motion? Exp Neurol 22: 13-25, 1968

Altman JA, Syka J, Shmigidina GN. Neuronal activity in the medial geniculate body of the cat during monaural and binaural stimulation. Exp Brain Res 10: 81-93, 1970.

Anstis S, Verstraten FA, Mather G. The motion aftereffect. Trends Cogn Sci 2: 111-117, 1998.

Baumgart F, Gaschler-Markefski B, Woldorff MG, Heinze HJ, Scheich H. A movement-sensitive area in auditory cortex. Nature 400: 724-726, 1999.

Blair RC, Karniski W. An alternative method for significance testing of waveform difference potentials. Psychophysiology 30: 518-524, 1993.

Blauert J. Spatial Hearing: the Psychophysics of Human Sound Localization. Cambridge, MA: MIT Press, 1997.

Boucher L, Lee A, Cohen YE, Hughes HC. Ocular tracking as a measure of auditory motion perception. J Physiol (Paris) 98: 235-248, 2004.

Bremmer F, Schlack A, Shah NJ, Zafiris O, Kubisckik M, Hoffman KP, Zilles K, Fink GR. Polymodal processing in posterior parietal and premotor cortex: a human fMRI study strongly implies equivalences between humans and monkeys. Neuron 29: 287-296, 2001.

Brunet D, Murray MM, Michel CM. Spatiotemporal analysis of multichannel EEG: CARTOOL. Comput Intell Neurosci 2011: 813870, 2011.

Chait M, Poeppel D, de Cheveigne A, Simon JZ. Human auditory cortical processing of changes in interaural correlation. J Neurosci 25: 8518-8527, 2005.

Delorme A, Makeig S. EEGLAB: an open source toolbox for analysis of single-trial EEG dynamics including independent component analysis. $J$ Neurosci Methods 134: 9-21, 2004.

Dong CJ, Swindale NV, Zakarauskas P, Hayward V, Cynader MS. The auditory motion aftereffect: its tuning and specificity in the spatial and frequency domains. Percept Psychophys 62: 1099-1111, 2000.

Dreyer A, Delgutte B. Phase locking of auditory-nerve fibers to the envelopes of high-frequency sounds: implications for sound localization. J Neurophysiol 96: 2327-2341, 2006.

Ducommun CY, Murray MM, Thut G, Bellmann A, Viaud-Delmon I, Clarke S, Michel CM. Segregated processing of auditory motion and auditory location: an ERP mapping study. Neuroimage 16: 76-88, 2002.

Ducommun CY, Michel CM, Clarke S, Adriani M, Seeck M, Landis T, Blanke O. Cortical motion deafness. Neuron 43: 765-777, 2004.

Ehrenstein WH. Auditory aftereffects following simulated motion produced by varying interaural intensity or time. Perception 23: 1249-1255, 1994.

Getzmann S, Lewald J. Effects of natural versus artificial spatial cues on electrophysiological correlates of auditory motion. Hear Res 259: 44-54, 2010.

Getzmann S, Lewald J. The effect of spatial adaptation on auditory motion processing. Hear Res 272: 21-29, 2011.

Getzmann S. Effect of auditory motion velocity on reaction time and cortical processes. Neuropsychologia 47: 2625-2633, 2009.

Getzmann S. Auditory motion perception: onset position and motion direction are encoded in discrete processing stages. Eur J Neurosci 33: 1339-1350, 2011.

Grantham DW, Wightman FL. Auditory motion aftereffects. Percept Psychophys 26: 403-408, 1979.

Grantham DW. Detection and discrimination of simulated motion of auditory targets in the horizontal plane. J Acoust Soc Am 79: 1939-1949, 1986.

Grantham DW. Motion aftereffects with horizontally moving sound sources in the free field. Percept Psychophys 45: 129-136, 1989.

Grantham DW. Adaptation to auditory motion in the horizontal plane: effect of prior exposure to motion on motion detectability. Percept Psychophys 52: 144-150, 1992.

Grantham DW. Auditory motion aftereffects in the horizontal plane: the effects of spectral region, spatial sector, and spatial richness. Acustica 84: 337-347, 1998

Grantham DW. Auditory motion perception: snapshots revisited. In: Binaural and Spatial Hearing in Real and Virtual Environments, edited by Gilkey T, Andersen TR. Mahwah, NJ: Lawrence Erlbaum, 1997, p. 295-313.

Grave de Peralta Menendez R, Gonzalez Andino S, Lantz G, Michel CM, Landis T. Noninvasive localization of electromagnetic epileptic activity. I. Method descriptions and simulations. Brain Topogr 14: 131-137, 2001

Grave de Peralta Menendez R, Murray MM, Michel CM, Martuzzi R, Gonzalez Andino SL. Electrical neuroimaging based on biophysical constraints. Neuroimage 21: 527-539, 2004. 
Griffiths TD, Bench CJ, Frackowiak RS. Human cortical areas selectively activated by apparent sound movement. Curr Biol 4: 892-895, 1994.

Griffiths TD, Rees A, Witton C, Shakir RA, Henning GB, Green GG. Evidence for a sound movement area in the human cerebral cortex. Nature 383: 425-427, 1996.

Griffiths TD, Rees A, Witton C, Cross PM, Shakir RA, Green GG. Spatial and temporal auditory processing deficits following right hemisphere infarction. A psychophysical study. Brain 120: 785-794, 1997.

Griffiths TD, Rees G, Rees A, Green GG, Witton C, Rowe D, Buchel C, Turner R, Frackowiak RS. Right parietal cortex is involved in the perception of sound movement in humans. Nat Neurosci 1: 74-79, 1998.

Griffiths TD, Green GG, Rees A, Rees G. Human brain areas involved in the analysis of auditory movement. Hum Brain Mapp 9: 72-80, 2000.

Grzeschik R, Bockmann-Barthel M, Muhler R, Hoffmann MB. Motiononset auditory-evoked potentials critically depend on history. Exp Brain Res 203: 159-168, 2010.

Guthrie D, Buchwald JS. Significance testing of difference potentials. Psychophysiology 28: 240-244, 1991.

Henning GB, Ashton J. The effect of carrier and modulation frequency on lateralization based on interaural phase and interaural group delay. Hear Res 4: 185-194, 1981.

Henning GB. Lateralization of low-frequency transients. Hear Res 9: 153172, 1983.

Koenig T, Melie-García L. A method to determine the presence of averaged event-related fields using randomization tests. Brain Topogr 23: 233-242, 2010.

Koenig T, Kottlow M, Stein M, Melie-Garcia L. Ragu: a free tool for the analysis of EEG and MEG event-related scalp field data using global randomization statistics. Comput Intell Neurosci 2011: 938925, 2011.

Kreitewolf J, Lewald J, Getzmann S. Effect of attention on cortical processing of sound motion: an EEG study. Neuroimage 54: 2340-2349, 2011.

Krumbholz K, Schonwiesner M, Rubsamen R, Zilles K, Fink GR, von Cramon DY. Hierarchical processing of sound location and motion in the human brainstem and planum temporale. Eur J Neurosci 21: 230-238, 2005

Kuwada S, Yin TC, Wickesberg RE. Response of cat inferior colliculus neurons to binaural beat stimuli: possible mechanisms for sound localization. Science 206: 586-588, 1979.

Lehmann D, Skrandies W. Reference-free identification of components of checkerboard-evoked multichannel potential fields. Electroencephalogr Clin Neurophysiol 48: 609-621, 1980.

Lewald J, Ehrenstein WH. The effect of eye position on auditory lateralization. Exp Brain Res 108: 473-485, 1996. [Erratum. Exp Brain Res 110: 322, 1996.].

Lewald J, Peters S, Corballis MC, Hausmann M. Perception of stationary and moving sound following unilateral cortectomy. Neuropsychologia 47: 962-971, 2009

Lewis JW, Beauchamp MS, DeYoe EA. A comparison of visual and auditory motion processing in human cerebral cortex. Cereb Cortex 10: 873-888, 2000.

Lu ZL, Sperling G. Attention-generated apparent motion. Nature 377: 237 239, 1995.

Lu ZL, Sperling G. Three-systems theory of human visual motion perception: review and update. J Opt Soc Am A Opt Image Sci Vis 18: 2331-2370, 2001.

Magezi DA, Krumbholz K. Evidence for opponent-channel coding of interaural time differences in human auditory cortex. J Neurophysiol 104: 1997-2007, 2010.

Mäkelä JP, McEvoy L. Auditory evoked fields to illusory sound source movements. Exp Brain Res 110: 446-454, 1996.

Malone BJ, Scott BH, Semple MN. Context-dependent adaptive coding of interaural phase disparity in the auditory cortex of awake macaques. $J$ Neurosci 22: 4625-4638, 2002.

Maris E, Oostenveld R. Nonparametric statistical testing of EEG- and MEG-data. J Neurosci Methods 164: 177-190, 2007.

Mather G, Verstraten F, Anstis SM. The Motion Aftereffect: A Modern Perspective. Cambridge, MA: MIT Press, 1998.

Mather G, Pavan A, Campana G, Casco C. The motion aftereffect reloaded. Trends Cogn Sci 12: 481-487, 2008.

Maunsell JH, van Essen DC. Functional properties of neurons in middle temporal visual area of macaque monkey. I. Selectivity for stimulus direction, speed, and orientation. J Neurophysiol 49: 1127-1147, 1983.

McAlpine D, Jiang D, Shackleton TM, Palmer AR. Responses of neurons in the inferior colliculus to dynamic interaural phase cues: evidence for a mechanism of binaural adaptation. J Neurophysiol 83: 1356-1365, 2000.
Michel CM, Murray MM, Lantz G, Gonzalez S, Spinelli L, Grave de Peralta R. EEG source imaging. Clin Neurophysiol 115: 2195-2222, 2004. Middlebrooks JC, Green DM. Sound localization by human listeners. Annu Rev Psychol 42: 135-159, 1991.

Murray MM, Brunet D, Michel CM. Topographic ERP analyses: a step-bystep tutorial review. Brain Topogr 20: 249-264, 2008.

Neelon MF, Jenison RL. The effect of trajectory on the auditory motion aftereffect. Hear Res 180: 57-66, 2003.

Neelon MF, Jenison RL. The temporal growth and decay of the auditory motion aftereffect. J Acoust Soc Am 115: 3112-3123, 2004.

Niedeggen M, Wist ER. The physiologic substrate of motion aftereffects. In: The Motion Aftereffect: A Modern Perspective, edited by Mather G, Verstraten F, Anstis S. Cambridge, MA: MIT Press, 1998, p. 125-155.

Niewenhuis S, Forstmann BU, Wagenmakers EJ. Erroneous analyses of interactions in neuroscience: a problem of significance. Nat Neurosci 14: 1105-1107, 2011.

Oldfield RC. The assessment and analysis of handedness: the Edinburgh inventory. Neuropsychologia 9: 97-113, 1971.

Oostenveld R, Fries P, Maris E, Schoffelen JM. FieldTrip: Open source software for advanced analysis of MEG, EEG, and invasive electrophysiological data. Comput Intell Neurosci 2011: 156869, 2011.

Pavani F, Macaluso E, Warren JD, Driver J, Griffiths TD. A common cortical substrate activated by horizontal and vertical sound movement in the human brain. Curr Biol 12: 1584-1590, 2002.

Perrin F, Pernier J, Bertrand O, Giard MH, Echallier JF. Mapping of scalp potentials by surface spline interpolation. Electroencephalogr Clin Neurophysiol 66: 75-81, 1987.

Picton TW, Bentin S, Berg P, Donchin E, Hillyard SA, Johnson Miller GA Jr, Ritter W, Ruchkin DS, Rugg MD, Taylor MJ. Guidelines for using human event-related potentials to study cognition: recording standards and publication criteria. Psychophysiology 37: 127-152, 2000.

Poirier C, Collignon O, Devolder AG, Renier L, Vanlierde A, Tranduly D, Scheiber C. Specific activation of the V5 brain area by auditory motion processing: an fMRI study. Brain Res Cong Brain Res 25: 650-658, 2005.

Rauschecker JP, Tian B. Mechanisms and streams for processing "what" and "where" in auditory cortex. Proc Natl Acad Sci U S A 97: 11800-11806, 2000.

Rauschecker JP, Romanski LM. Auditory cortical organization: evidence for functional streams. In: The Auditory Cortex, edited by Winer JA, Schreiner C. New York: Springer, 2011, p. 99-116.

Rosenthal R, Gaito J. The interpretation of levels of significance by psychological researchers. J Psychol 55: 33-38, 1963.

Rosnow RL, Rosenthal R. Statistical procedures and the justification of knowledge in psychological science. Am Psychol 44: 1276-1284, 1989.

Schögl A, Keinrath C, Zimmerman D, Scherer R, Leeb R, Pfurtscheller G. A fully automated correction method for EOG artifacts in EEG recordings. Clin Neurophysiol 118: 98-104, 2007.

Schreiner CE, Kanold PO, Ojima H, Shamma SA, Lomber SG. Auditory cortical function: insights from current approaches. Acoust Today 8: 42-50, 2012

Scott BH, Malone BJ, Semple MN. Representation of dynamic interaural phase difference in auditory cortex of awake rhesus macaques. $J$ Neurophysiol 101: 1781-1799, 2009.

Skrandies W. The effect of stimulation frequency and retinal stimulus location on visual evoked potential topography. Brain Topogr 20: 15-20, 2007.

Smith KR, Okada K, Saberi K, Hickok G. Human cortical auditory motion areas are not motion selective. Neuroreport 15: 1523-1526, 2004.

Smith KR, Saberi K, Hickok G. An event-related fMRI study of auditory motion perception: no evidence for a specialized cortical system. Brain Res 1150: 94-99, 2007.

Sovijärvi AR, Hyvärinen J. Auditory cortical neurons in the cat sensitive to the direction of sound source movement. Brain Res 73: 455-471, 1974.

Spitzer MW, Semple MN. Interaural phase coding in auditory midbrain: influence of dynamic stimulus features. Science 254: 721-724, 1991.

Spitzer MW, Semple MN. Responses of inferior colliculus neurons to time-varying interaural phase disparity: effects of shifting the locus of virtual motion. J Neurophysiol 69: 1245-1263, 1993.

Stanley RM, Matthews N. Invalid cues impair auditory motion sensitivity. Perception 32: 731-740, 2003.

Stumpf E, Toronchuk JM, Cynader MS. Neurons in cat primary auditory cortex sensitive to correlates of auditory motion in three-dimensional space. Exp Brain Res 88: 158-168, 1992.

Talairach J, Tournoux P. Co-Planar Stereotaxic Atlas of the Human Brain. Stuttgart, Germany: Thieme, 1988. 
Thompson P, Burr D. Visual aftereffects. Curr Biol 19: R11-R14, 2009.

Toronchuk JM, Stumpf E, Cynader MS. Auditory cortex neurons sensitive to correlates of auditory motion: underlying mechanisms. Exp Brain Res 88: $169-180,1992$

Tzovara A, Murray MM, Bourdaud N, Chavarriaga R, Millan R, De Lucia M. The timing of exploratory decision-making revealed by singletrial topographic EEGanalyses. Neuroimage 60: 1959-1969, 2012.

Vaughan HG Jr. The neural origins of human event-related potentials. Ann N Y Acad Sci 388: 125-138, 1982.

Wagner H, Kautz D, Poganiatz I. Principles of acoustic motion detection in animals and man. Trends Neurosci 20: 583-588, 1997.

Warren JD, Zielinski BA, Green GG, Rauschecker JP, Griffiths TD. Perception of sound-source motion by the human brain. Neuron 34: 139148, 2002.
Weliky M, Bosking WH, Fitzpatrick D. A systematic map of direction preference in primary visual cortex. Nature 379: 725-728, 1996.

Wightman FL, Kistler DJ. The dominant role of low-frequency interaural time differences in sound localization. J Acoust Soc Am 91: 1648-1661, 1992.

Xiang J, Chuang S, Wilson D, Otsubo H, Pang E, Holowka S, Sharma R, Ochi A, Chitoku S. Sound motion evoked magnetic fields. Clin Neurophysiol 113: 1-9, 2002.

Yin TC, Kuwada S. Binaural interaction in low-frequency neurons in inferior colliculus of the cat. II. Effects of changing rate and direction of interaural phase. J Neurophysiol 50: 1000-1019, 1983.

Zimmer U, Macaluso E. Interaural temporal and coherence cues jointly contribute to successful sound movement perception and activation of parietal cortex. Neuroimage 46: 1200-1208, 2009. 